\title{
Voice Controlled Wheel Chair for Persons with Disability
}

\section{Dr. Rohith S1, Devireddy Brijesh Kumar Reddy ${ }^{2}$, CharanKumar H N ${ }^{3}, \mathrm{HL}$ Tejavardhan ${ }^{4}$, B Hanumanth Reddy ${ }^{5}$}

${ }^{1}$ Professor, Department of Electronics and communication Engineering, Nagarjuna College of Engineering and

Technology, Bangalore, Karnataka, India

2, 3, 4, 5B. E. Student, Department of Electronics and communication Engineering, Nagarjuna College of Engineering and Technology, Bangalore, Karnataka, India

\section{ABSTRACT}

Article Info

Volume 8, Issue 3

Page Number : 572-577

\section{Publication Issue}

May-June-2021

\section{Article History}

Accepted : 03 June 2021

Published : 08 June 2021
Many disabled people usually rely on others in their standard of living especially in getting from one place to a different. To use wheelchair, they have someone to assist them in moving the wheelchair. Having a wheelchair system will help handicapped persons become independent. The system could be a wireless wheelchair system with a voice recognition system for triggering and controlling all its movements. The wheelchair responds to the voice command by user to perform any movement. It integrates a microcontroller, android phone, control interface board to maneuver the wheelchair. By using the system, users are able to operate the wheelchair by simply talking to wheelchair microphone. the essential movement functions includes forward and reverse direction, left and right turns and stop. It utilizes Renesas Microcontroller to manage the system operations. It communicates with the voice recognition processor to detect word spoken and so determines the corresponding output command to drive the left and right motors. To accomplish this task, a programming language program is written and stored within the controller's memory. GSM module is employed to send an alert message to their care takers just in case of emergency situations.

Keywords : Wheelchair, voice-recognition system, Renesas Controller, GSM module.

\section{INTRODUCTION}

A mortal with locomotive disabilities needs a wheelchair to perform functions that need a person to maneuver around. Person can do so manually by pushing the wheelchair along with his hands. However, a larger number of individuals have weak upper limbs or find the manual mode of operating too tiring. Hence, it's desirable to produce them with a wheelchair that may be controlled by moving a joystick or through voice commands. All this could be achieved at a price that's affordable for as many handicapped people as possible, also as for organizations that support them. With these 
requirements in mind, we propose an automatic wheelchair that can be operated through voice commands. Through research and style-wise, the ability wheelchair to regulate development along with safe and effective use of the availability independence and self-use mobility. This project describes a

\section{LITERATURE SURVEY}

Tongue Drive system (TDS) is a tongue-operated unobtrusive wireless assistive technology. It translates users' intentions into control commands by detecting and classifying their voluntary tongue motion utilizing a small permanent magnet, secured on the tongue, and an array of magnetic sensors mounted on a headset outside the mouth or an orthodontic brace inside. Here they used hall effect sensors, HT12 encoder and decoder transmitter and receiver, AT mega 328p microcontroller. There are three hall effect sensors used in this project which are going to be decide in which direction the wheel chair has to move and micro controller controls the motors through a set of programs which is previously dumped.

A voice controller and touch screen machine which guides the wheel chair by the voice of a person. In this project they used touch screen sensor, microphone, voice recognition module, driver circuit. The wheel chair contains two modes of operation touch screen and voicecontrolled system. We can use only one mode of operation i.e., either voice control or touch screen. The touch screen used here is 5- wire resistive type consists of six quadrants. In addition, a break control switch is used to stop the wheel chair.

Gesture Controlled Wheelchair for Quadriplegic Children. This involves the use of micro electromechanical sensors, micro-controllers, transmitter-receiver pair for wireless transmission of data and the wheelchair for the prototype. The wheel chair moves according to the hand movements as we place the transmitter part in the hand. The MEMS wheelchair that is controlled by using the user's voice only. This project aims to facilitate the movement of disabled people and elderly those who cannot move properly then enable them to steer better lives with no problem.

Sensor is inside the transmitter. ADXL335 gives output in analog voltage form based on the inclination of the sensor and then it converts to the digital. Then it encoded and send to the decoder through the transmitter and receiver and then it is passed to the controller and according to received signal then microcontroller controls the motors through motor driver.

Fire Alarm system based on GSM network. They used components like smoke sensor, temperature sensor, sound light alarm module, MSP430F149 Micro controller and GSM wireless communication module TC35I. In this system smoke sensor and temperature sensor will identify the fire catching and sends to the MSP430 Micro controller and then the controller activates the light sound module and sends the signal to the TC35I module and the message will be sent to the user's phone and they will act accordingly.

Control System of powered Wheelchairs based on tongue motion detector. Here the tongue motion is detected with several vibration film sheets that are embedded in the headset. The sensor used is piezoelectric film it detects the motion of tongue and sends to microcontroller1 which converts the analog signal to digital signal and to receiving microcontroller-2 through NRF24L0 transmitter and receiver. Then the microntroller-2 will control motors of wheelchair which is connected by the motor driver according to the received digital signals.

\section{SYSTEM REQUIREMENTS}

\section{Hardware Components:}

GSM Module (SIM 900A)

Renesas Microcontroller (RL78)

Motor Driver (L293D) 
High Power 12v DC Motor

Battery

Wheelchair

Android Mobile

ALCD

\section{Software Requirements:}

Cube Suite Plus

Android Studio

\section{GSM Module (SIM 900A):}

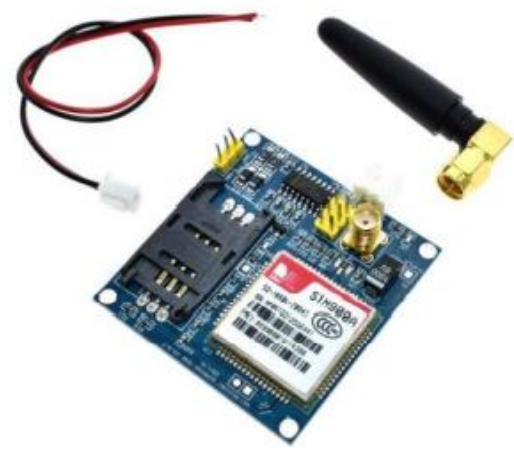

Fig.3.1 GSM module

GSM module uses mobile telephone technology to provide a data link to a remote network. GSM module is uniquely identified in a network by the SIM inserted in it. In the view of a mobile user GSM is similar to an ordinary mobile. Data is sent and receives data via radio waves. It used TDMA technique with eight radio timeslots grouped into a TDMA frame.

\section{Renesas Microcontroller (RL78)}

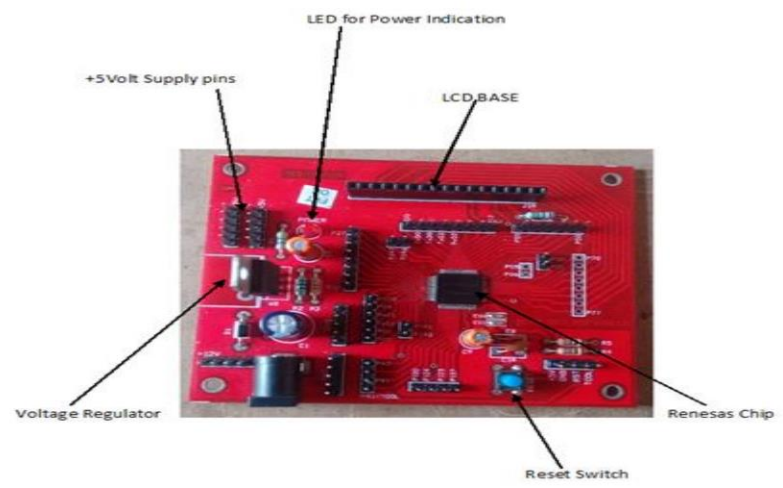

Fig.3.2 Renesas Microcontroller
Renesas is a 16-bit microcontroller which surpasses its predecessor i.e., 8051 family of microcontrollers, with various in-built features such as:

- ROM: 64kb KB, RAM: 4 KB, Data flash memory: 4 $\mathrm{KB}$

- On-chip high-speed on-chip oscillator

-On-chip single power supply flash memory (with prohibition of block erase/writing function).

- On-chip debug function.

- General-purpose register: 8 bits $\times 32$ registers $(8$ bits $\times 8$ registers $\times 4$ banks).

In total there are 11 ports with 58 Input/Output Pins in this microcontroller.

\section{Motor Driver (L293D):}

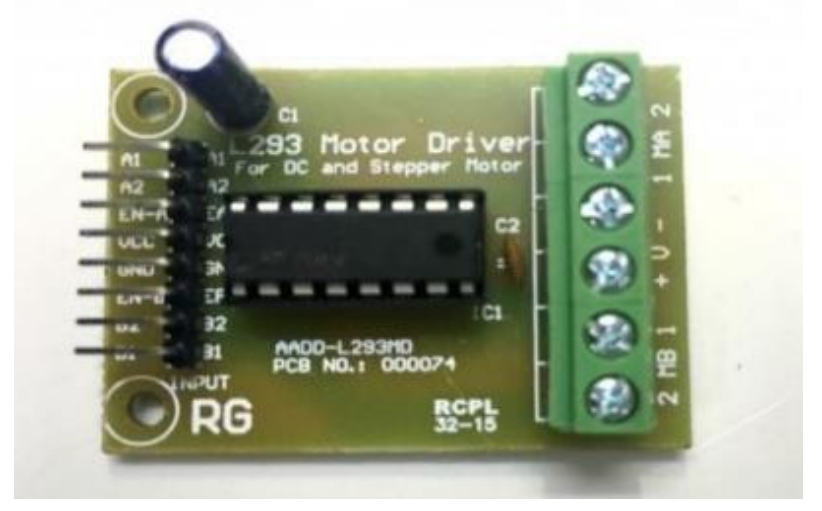

Fig.3.3. Motor Driver

The L293D is a dual-channel H-Bridge motor driver which can drive a pair of DC motors or one stepper motor. It can individually drive up to two motors making it suitable for building two wheel robot platforms. Using the direction control pins, we can control whether the motor spins forward or backward. The L293 is limited to $600 \mathrm{~mA}$, but in reality, can only handle much small currents unless some heat sinking is done to keep the case temperature down.

\section{DC Motor:}

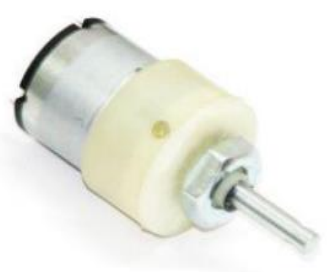

Fig.3.4. DC Motor 
NR-DC-ECO is high-quality low-cost DC geared motor. it's product of Brass gears and steel pinions to make sure longer life and better wear and tear properties. The gears are anchored to hardened steel spindles. These spindles are polished to a mirror finish. For silent running these spindles rotate between bronze plates. The entire assembly is roofed with a plastic ring. All the bearings are permanently lubricated and so require no maintenance. Inside the gear box the motor is screwed to to it.

\section{ALCD:}

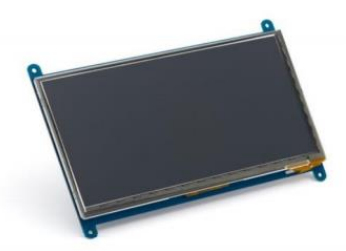

Fig.3.5. ALCD

Alpha numeric LCD (ALCD) is used to display information about project. The LCD used is $16 \times 2,2$ rows and 16 columns. In each row we can display 16 characters. The 1-byte data line of lcd is connected to the Port 7.0 to Port 7.7 of the microcontroller. The enable pin of lcd is connected to the Port 0.6 of the microcontroller. The RS pin of the lcd is connected to the Port 0.5 of the microcontroller. Pin 1 of lcd is $+5 \mathrm{~V}$ power supply, pin 2 is GND, pin 3 is for contrast adjustment, pin 5 is for read or write operation. So, pin 5 is GND. Pin 15 is the backlight led anode terminal connected to $+5 \mathrm{~V}$ and pin 16 is backlight led cathode terminal connected to GND.

Cube Suite Plus: For Programming the Controller Operations

Android Studio: For developing the Android App

\section{METHODOLOGY}

This project describes a smart wheelchair that may be controlled by using the android application and the person who is operating the wheelchair his voice also. The most aim of this project is to facilitate the movement of the disabled people and elderly folks that cannot move properly so with this we are able to enable them to guide better lives with none problem. This project includes two parts which are software and hardware. it's realized that for input of human voice we are using Android phone as an intermediary. During this project Renesas controller is employed as controller to regulate the movement of wheelchair supported the human voice as an input from android mobile.

There are usually five conditions of the wheelchair will be described as the following:
i. Moving forward
ii. Moving backward
iii. Turning right
iv. Turning left
v. Stop

\section{BLOCK DIAGRAM}

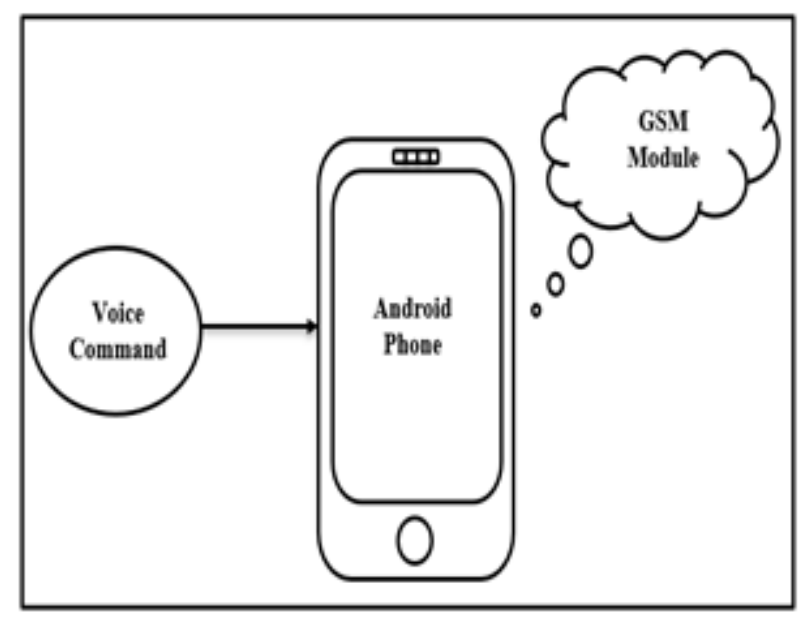

Fig. 5.1 : Transmission block

This device has mainly two blocks Transmission Block and Reception Block.

1. The Transmission Block contains Android phone which is connected with Google Assistant and GSM Module. Here the patient controls the wheel chair though some commands like STOP, MOVE 
FORWARD, TURN RIGHT, INCREASE SPEED, DECREASE SPEED like etc. These commands are given by the patient by Voice commands to Android Phone which has Google Assistant and android application which is made through the java programming which is already installed in it. The Android Phone is connected to the Renesas Controller Wirelessly by the help of GSM Module.

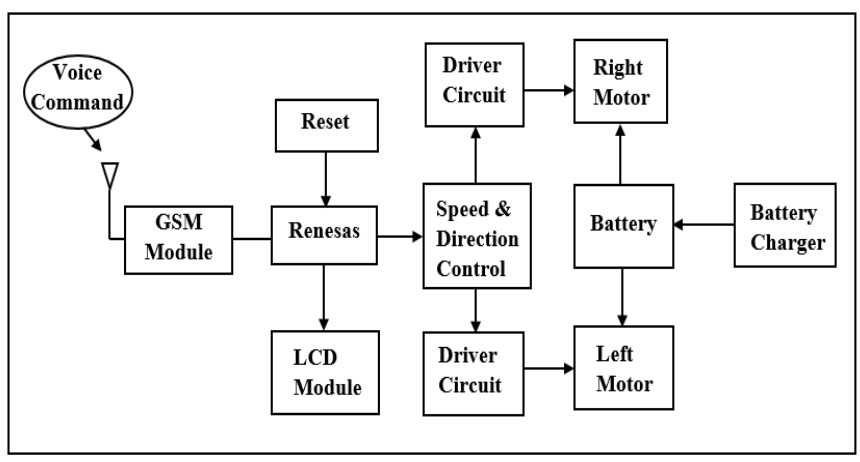

Fig.5.2 : Reception Block

2. In Reception block there are Renesas Controller which is connected with the android through GSM Module, Speed and direction Block Control, Driver Circuit, Battery, High Power 12V DC Motors, LCD, Battery and Battery Charger. The commands from the Android Phone are sent to the GSM Module through

\section{RESULTS}

A wheel chair with voice control is modelled with an android app to recognize the voice commands. Specific commands such as Forward, Backward etc., that are dumped in the microcontroller will be considered for moving the wheel chair.

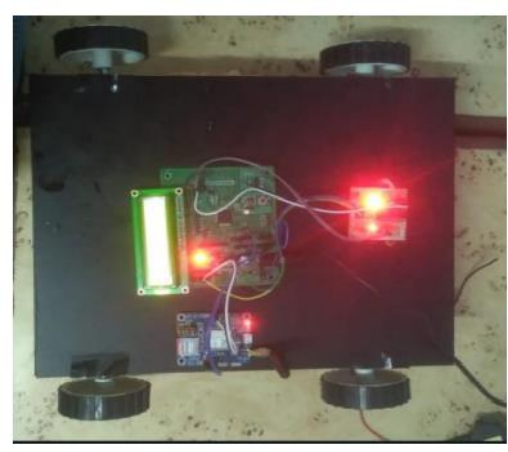

Fig.6.1: Assembled Prototype

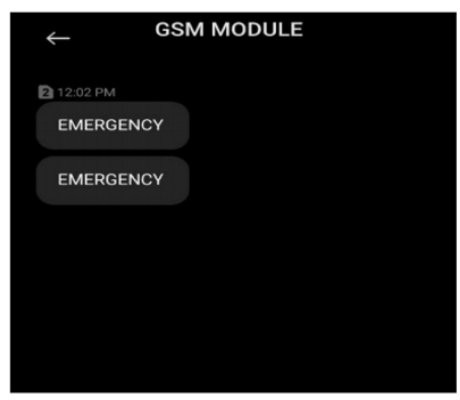

Fig.6.2: Text messages received from GSM module

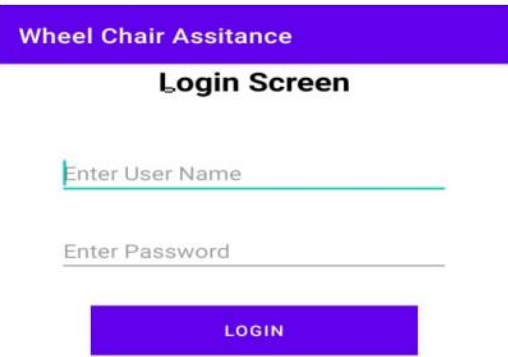

Fig.6.3: Login Screen

\section{Welcome Admin}

\section{CARE TAKER NUMBER}

\section{VOICE INPUT}

Fig.6.4 Welcome screen

\section{Register Care Taker Number}

\section{UPDATE}

Fig.6.5 Registration of care taker Number 


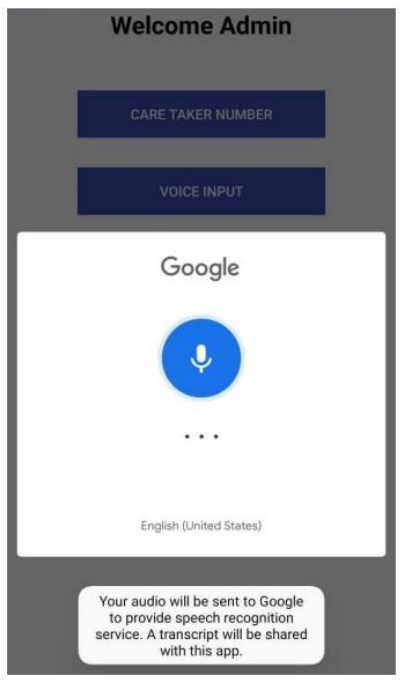

Fig.6.6 Speech Recognition via Number Google Assistant

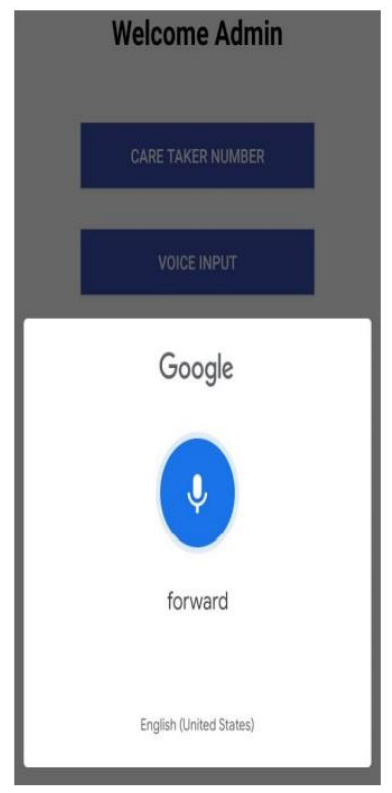

Fig.6.7 Giving voice command to Google Assistant

\section{Welcome Admin}

\section{CARE TAKER NUMBER}

\section{VOICE INPUT}

forward

SMS Sent Successfully

Fig.6.8 SMS successfully sent to GSM Module

\section{VII.CONCLUSION}

This wheel chair is designed and constructed for paralysed persons. The motor drive and control system of this prototype intelligent wheelchair has been presented. The proposed voice operated intelligent wheelchair would bring more convenience for the disabled people. The proposed model uses Android app which makes it easy to use. Emergency mode ensures more security by alerting the caretaker by sending an alert message to mobile phone of the person who is registered as a caretaker in the app.

\section{REFERENCES}

[1]. Amrita Mahadev Chavan, Bhagyashri Balkrishna Patil, Sayali Balaso Jadhav, Priyanka Dinkar Yadav, "Wheel chair operated by Tongue Motion", International Journal of Advanced Research in Electronics and Communication Engineering (IJARECE) Volume 5, Issue 2, February 2016.

[2]. Mohammed FaeikRuzaij, S. Poonguzhali, "Design and Implementation of Low-Cost Intelligent Wheelchair", International Conference on Recent Trends in Information Technology, 2012.

[3]. Yash M. Jain, Saurabh S. Labde, Dr. Sunil Karamchandani, "Gesture Controlled Wheelchair for Quadriplegic Children", The 2016 3rd International Conference on Systems and Informatics (ICSAI 2016).

[4]. Masato Nishimori, Takeshi Saitoh and Ryosuke Konishi, "Voice Controlled Intelligent Wheelchair", SICE Annual Conference 2007 Sept. 17-20, 2007.

[5]. Lu Liao, Ying Wu, Yi Xiang, Xinping Yan, "Control System of powered Wheelchairs based on tongue motion detector", 2016 15th International Conference on Cognitive 
informatics and Cognitive Computing, August

2016.

\section{Cite this article as :}

Dr. Rohith S, Devireddy Brijesh Kumar Reddy, CharanKumar H N, HL Tejavardhan, B Hanumanth Reddy, " Voice Controlled Wheel Chair for Persons with Disability", International Journal of Scientific Research in Science and Technology(IJSRST), Print ISSN : 2395-6011, Online ISSN : 2395-602X,Volume 8, Issue 3, pp.572-577, May-June-2021. Available at doi : https://doi.org/10.32628/IJSRST2183125 Journal URL : https://ijsrst.com/IJSRST2183125 